\title{
A 3D Model of Magnetohydrodynamic Voltages: Comparison with Voltages Observed on the Surface ECG during Cardiac MRI
}

\author{
GM Nijm ${ }^{1}$, S Swiryn ${ }^{1,2}$, AC Larson ${ }^{1}$, AV Sahakian ${ }^{1}$ \\ ${ }^{1}$ Northwestern University, Evanston, IL, USA \\ ${ }^{2}$ HeartCare Midwest, Peoria, IL, USA
}

\begin{abstract}
The magnetohydrodynamic (MHD) effect generates voltages which distort the ECG obtained during cardiac MRI. Consequently, MHD voltages result in triggering problems for MR image acquisition. In addition, since the MHD effect is related to blood flow, analysis of it not only as interference, but also as a signal may provide useful blood flow information. Comsol Multiphysics modeling software was used to compute and model the $M H D$ voltages in $3 D$. These voltages were compared with $M H D$ voltages obtained experimentally from the subtraction of ECGs taken outside the MRI magnet from ECGs taken inside the magnet. The maximum MHD voltage magnitude for the experimental data was $0.2 \mathrm{mV}$ and was $3.04 \mathrm{mV}$ for the modeled data when calculated on the surface of the uniform volume conductor. By modeling $M H D$ voltages in $3 D$, we can learn about their effect on the ECG during cardiac MRI.
\end{abstract}

\section{Introduction}

As described by the magnetohydrodynamic (MHD) effect, voltages generated by blood flow in the body may be observed on the surface electrocardiogram (ECG) during cardiac magnetic resonance imaging (MRI) $[1,2]$. The largest MHD voltage magnitude is observed orthogonal to the magnetic field lines and the direction of fluid flow. MHD voltages distort the ECG and result in triggering problems for MR image acquisition $[3,4]$. In addition, these voltages are related to flow, so examination of the MHD effect can provide useful blood flow information.

The MHD voltage (V) may be described by a simplified equation, shown below:

$$
\mathbf{V}=\int_{0}^{L} \boldsymbol{u} \times \boldsymbol{B} \cdot d \boldsymbol{L}
$$

where $\mathbf{B}$ is the magnetic flux density, $\mathbf{u}$ is the velocity of the fluid, and $\mathbf{L}$ is the distance vector between electrodes.
This simplified equation makes the assumption of a uniform velocity profile; however, the velocity of blood typically follows a parabolic profile.

The equations which completely describe these phenomena involve the coupling of Maxwell's equations and Navier-Stokes equations. Maxwell's equations may be formulated as Maxwell-Ampère's law (2), Faraday's law (3), and the two forms of Gauss' law $(4,5)$ :

$$
\begin{gathered}
\nabla \times \mathbf{H}=\mathbf{J}+\frac{\partial \mathbf{D}}{\partial t} \\
\nabla \times \mathbf{E}=-\frac{\partial \mathbf{B}}{\partial t} \\
\nabla \cdot \mathbf{D}=\rho \\
\nabla \cdot \mathbf{B}=0
\end{gathered}
$$

where $\mathbf{H}$ is the magnetic field intensity, $\mathbf{J}$ is the current density, $\mathbf{D}$ is the electric flux density, $\mathbf{E}$ is the electric field intensity, $\mathbf{B}$ is the magnetic flux density, and $\rho$ is the electric charge density [5]. Incompressible Navier-Stokes equations may be formulated as the momentum balance equation (6) and the equation of continuity (7) :

$$
\begin{gathered}
\rho \frac{\partial \mathbf{u}}{\partial t}-\eta \nabla^{2} \mathbf{u}+\rho(\mathbf{u} \cdot \nabla) \mathbf{u}+\nabla p=\mathbf{F} \\
\nabla \cdot \mathbf{u}=0
\end{gathered}
$$

where $\rho$ is the density, $\mathbf{u}$ is the velocity field, $\eta$ is the dynamic viscosity, $p$ is the pressure, and $\mathbf{F}$ is the volume force vector. Due to the complexity of these equations, finite element methods are typically used to numerically compute MHD voltages.

\section{Methods}

\subsection{Model methods}

Comsol Multiphysics ${ }^{\mathrm{TM}}$ modeling software (version 3.3a), which implements the finite element method to 
solve partial differential equations, was used to create a three-dimensional model of blood flow through a blood vessel. The model geometry consisted of a vessel with an inner radius of $1.0 \mathrm{~cm}$ and length of $3.0 \mathrm{~cm}$, oriented along the $\mathrm{x}$-axis of the $3 \mathrm{D}$ Cartesian coordinate axes. The blood vessel wall had a thickness of $2.2 \mathrm{~mm}$, making the outer radius of the blood vessel equal to $1.22 \mathrm{~cm}$. The vessel was surrounded by a cylindrical uniform volume conductor with a radius of $20 \mathrm{~cm}$ and length of $3.0 \mathrm{~cm}$.

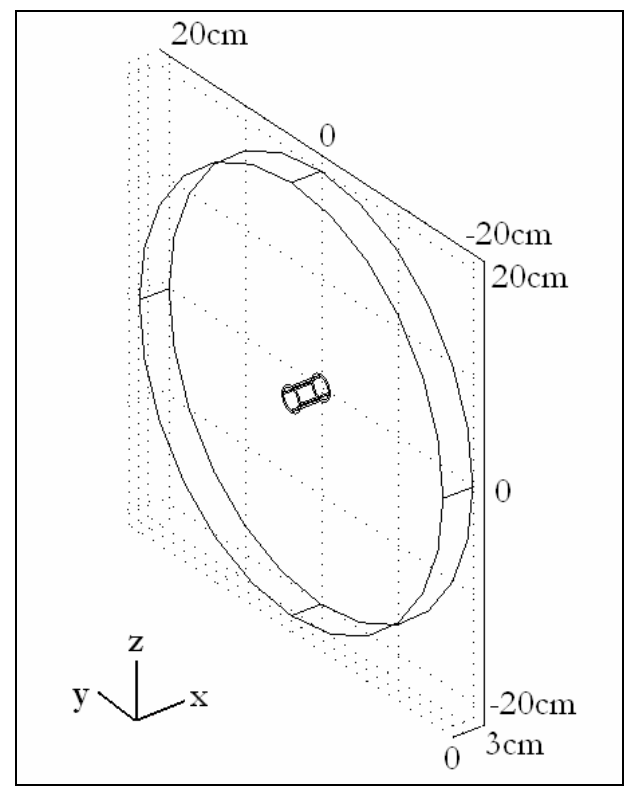

Figure 1: Geometry of the model consisting of a blood vessel surrounded by a uniform volume conductor.

A 3.0 Tesla static magnetic field was simulated to represent the large magnetic field generated by the MRI magnet. The magnetic field was oriented such that the magnetic field lines ran parallel to the z-axis.

Physiologically realistic values were used for the properties of blood, the blood vessel wall, and the uniform volume conductor, which are shown in Table 1.

\begin{tabular}{|l|l|}
\hline Parameter & Value \\
\hline Density of blood & $1050 \mathrm{~kg} / \mathrm{m}^{3}$ \\
\hline Dynamic viscosity of blood & $0.004 \mathrm{~Pa} \cdot \mathrm{s}$ \\
\hline Blood conductivity & $0.5 \mathrm{~S} / \mathrm{m}$ \\
\hline Vessel wall conductivity & $0.15 \mathrm{~S} / \mathrm{m}$ \\
\hline Uniform volume conductor conductivity & $0.2 \mathrm{~S} / \mathrm{m}$ \\
\hline
\end{tabular}

Table 1: Model parameters for blood, blood vessel wall, and uniform volume conductor [6].

A parabolic velocity profile was implemented, and the velocity varied over time to simulate pulsatile flow. The equation used for input velocity $\mathrm{v}$ is expressed as: $v(y, z, t)=\left(\frac{R^{2}-y^{2}-z^{2}}{R^{2}}\right) *\left(3 e^{-6 t} \sin (2 \pi t)\right)$

where $\mathrm{R}$ is the inner radius of the vessel, and the velocity of the fluid is in the $\mathrm{x}$-direction.

Maxwell's equations, which quantify electromagnetics, and Navier-Stokes equations, which quantify fluid dynamics, were coupled to generate MHD voltages. The resulting MHD voltages were computed by the finite element method using an iterative solver (GMRES) and were modeled in 3D. The model was solved for $50 \mathrm{~ms}$ time steps from 0.0 to 1.0 seconds. The mesh used to compute the solution consisted of 2809 elements.

\subsection{Experimental methods}

The dimensions of the vessel and the properties of the blood were chosen to be similar to those in the segment of the aortic arch which is oriented in the sagittal direction, in order to compare the magnitudes of the MHD voltages computed by the model with experimentally acquired data from ECG during cardiac MRI. These data resulted from the subtraction of ECGs taken from outside the MRI magnet (undistorted by MHD) from ECGs taken from inside the magnet (distorted by MHD) from nine healthy subjects [7]. The resulting signal was identified as the MHD signal, since the difference between these two signals was the MHD voltage. In this experiment, the electrodes were oriented in the transverse direction (shown in Figure 2), and subjects were oriented in the supine and prone positions, so MHD voltages resulted primarily from blood flow in the segment of the aortic arch oriented in the sagittal direction. The magnitude and duration of these MHD signals were quantified. Also in Figure 2, the theoretical electrode locations to obtain the voltages measured in the model are indicated.

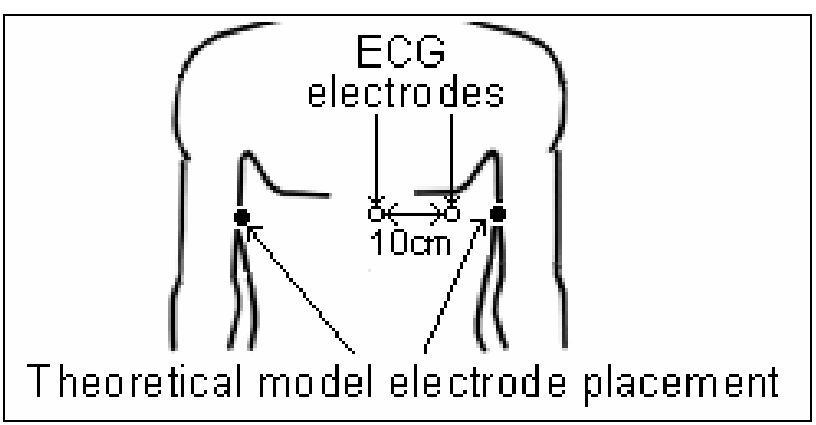

Figure 2: Experimental ECG electrode placement and theoretical locations on the body where the electrodes would be placed to obtain the MHD voltages that are measured in the model. 


\section{Results}

\subsection{Model MHD voltages}

The resulting model showed that the greatest magnitude voltages resulting from the MHD effect occurred orthogonal to the magnetic field lines and direction of fluid flow, as expected. In Figure 3, these voltages are shown on the input face in the $y-z$ plane for a series of time steps. The maximum voltage difference was observed in the y-direction, with a value of $31.2 \mathrm{mV}$ when measured directly on the surface of the blood, 24.0 $\mathrm{mV}$ when measured on the blood vessel walls, and 3.04 $\mathrm{mV}$ when measured from the outer surface of the uniform volume conductor.

In addition, the MHD voltages varied as the velocity changed over time. Of the calculated time steps, the maximum MHD voltage occurred at $\mathrm{t}=0.15$ seconds with a value of $3.04 \mathrm{mV}$, and the minimum voltage occurred at $\mathrm{t}=0.65$ seconds with a value of $-0.152 \mathrm{mV}$. The MHD voltages were zero at $0.0,0.5$, and 1.0 seconds. The MHD voltages observed from the outer radius of the uniform volume conductor on the input face are plotted vs. time in Figure 4 for all time steps from 0.0 to 1.0 seconds.

\subsection{Comparison with experimental data}

When compared with the experimentally obtained MHD signals, the amplitudes of the resulting signals differed by a single order of magnitude. Namely, the mean of the maximum MHD voltages observed from the experimental values was $0.2 \mathrm{mV}$, and the maximum from the model was $3.04 \mathrm{mV}$, as observed from the input face from the outer surface of the uniform volume conductor.

\section{Discussion and conclusions}

There are several reasons why the MHD voltage magnitudes obtained experimentally differ from those obtained from the model. First, the model geometry is very simplified. Second, the model is comprised of only the vessel and a uniform volume conductor, while there are many other structures which are present in the human between the heart and the surface, such as the lungs, ribs, etc. Third, the experimental ECG electrode placement differed from the theoretical electrode placement for the model. Future work will improve the accuracy of the geometry and include other anatomical structures in the model in order to produce more physiologically realistic MHD voltages, since a uniform volume conductor cannot adequately represent the physical characteristics of the different tissues which separate the heart from the body's surface.

For the experiment, blood flow from other vessels and the heart itself may also have contributed to the MHD voltages observed on the surface ECG; however, the most significant contribution of blood flow is from the aortic arch due to the velocity of blood, vessel diameter, and orientation of this vessel. Consequently, the model was chosen to have parameters similar to those in the sagittal segment of the aortic arch for comparison with the experimental data.

In conclusion, by modeling MHD voltages in $3 \mathrm{D}$, we can quantify the distortion of the ECG during cardiac MRI, which may eventually lead to diagnostically useful ECGs as well as the capability to provide additional information about blood flow.

\section{Acknowledgements}

This material is based upon work supported under a National Science Foundation Graduate Research Fellowship. in part by National Institutes of Health grant number NHLBI HL079148, and in part by a grant from the Dr. Scholl Foundation.

\section{References}

[1] Togawa T, Okai O, Oshima M. Observation of blood flow e.m.f. in externally applied strong magnetic field by surface electrodes. Med Biol Eng 1967 Mar;5(2):169-70.

[2] Dimick RN, Hedlund LW, Herfkens RJ, Fram EK, Utz J. Optimizing electrocardiograph electrode placement for cardiac-gated magnetic resonance imaging. Invest Radiol 1987 Jan;22(1):17-22.

[3] Tenforde TS. Magnetically induced electric fields and currents in the circulatory system. Prog Biophys Mol Biol 2005 Feb-Apr;87(2-3):279-88

[4] Tenforde TS, Gaffey CT, Moyer BR, Budinger TF. Cardiovascular alterations in Macaca monkeys exposed to stationary magnetic fields: experimental observations and theoretical analysis. Bioelectromag 1983;4(1):1-9.

[5] COMSOL Multiphysics ${ }^{\mathrm{TM}}$ Software and Manuals, www.comsol.com.

[6] Kinouchi Y, Yamaguchi H, Tenforde TS. Theoretical analysis of magnetic field interactions with aortic blood flow. Bioelectromagnetics 1996:17(1);21-32.

[7] Nijm GM, Swiryn S, Larson AC, Sahakian AV. Characterization of the magnetohydrodynamic effect as a signal from the surface electrocardiogram during cardiac magnetic resonance imaging. Computers in Cardiology 2006;33:269-72.

Address for correspondence:

Prof. Alan V. Sahakian

EECS and BME Departments

Northwestern University

2145 Sheridan Road

Evanston, IL 60208

USA

sahakian@ece.northwestern.edu 


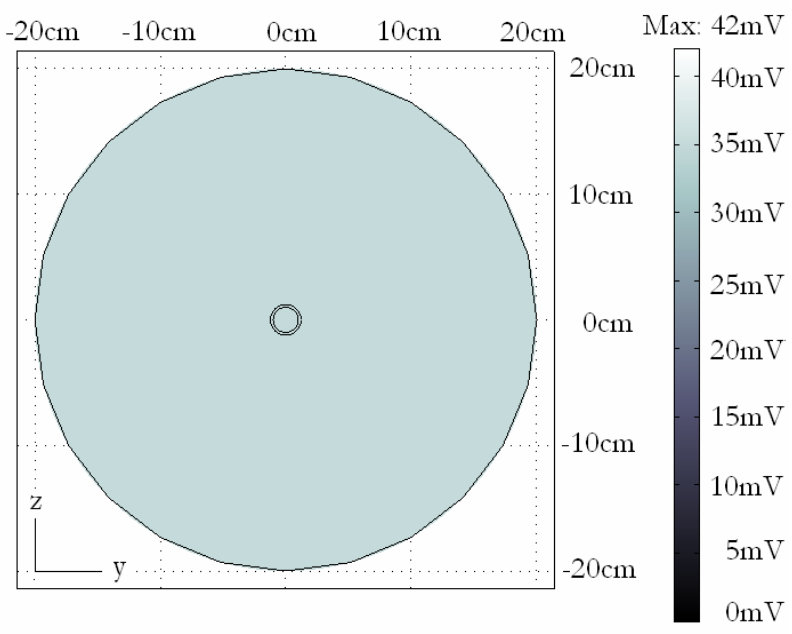

(a) $\mathrm{t}=0.0$ seconds

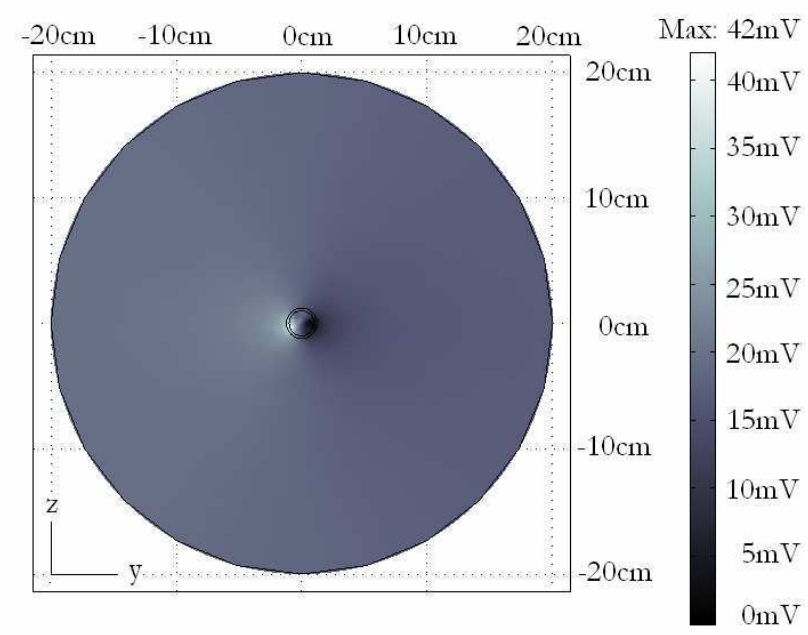

(b) $\mathrm{t}=0.15$ seconds

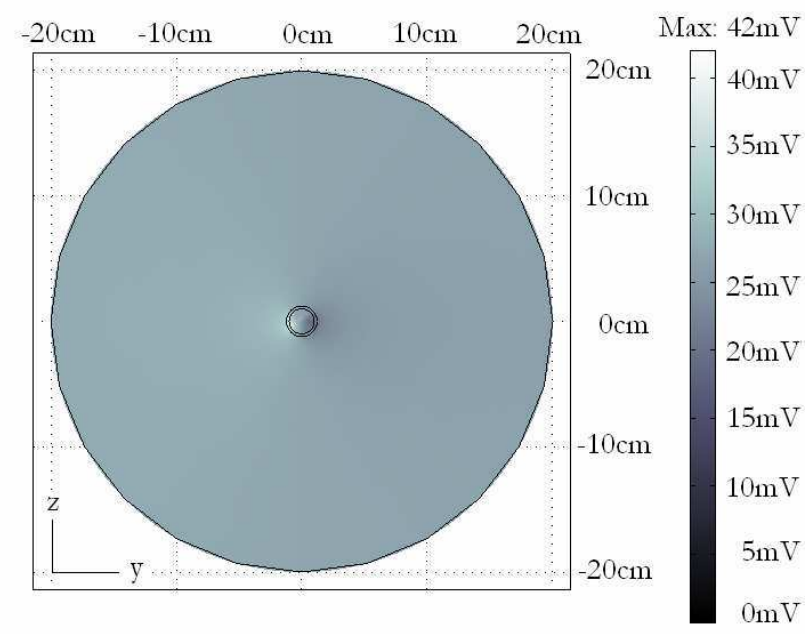

(c) $\mathrm{t}=0.3$ seconds

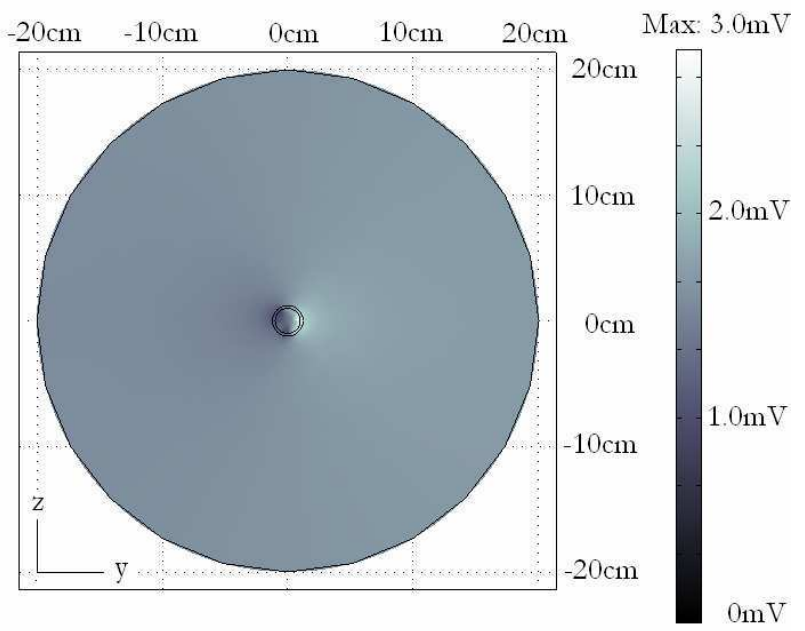

(d) $\mathrm{t}=0.65$ seconds

Figure 3: MHD voltages observed from the input face in the $y-z$ plane for four time steps. The bar to the right of each figure shows the color scale corresponding to the different values of voltages. Note the different scale for (d) where $\mathrm{t}=0.65$ seconds; this time step occurs during retrograde flow, where voltages are negative and of relatively small magnitude, which requires a different scale.

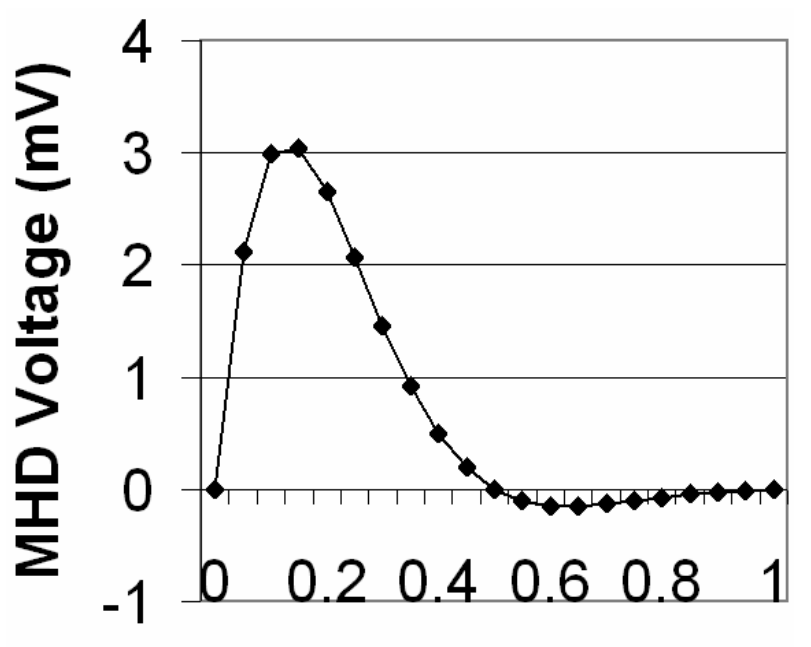

\section{Time (seconds)}

Figure 4: Plot of maximum MHD voltages vs. time observed from the volume conductor outer edge on the input face. Of the calculated time steps, the maximum MHD voltage occurred at $\mathrm{t}=0.15$ seconds with a value of $3.04 \mathrm{mV}$, and the minimum voltage occurred at $\mathrm{t}=0.65$ seconds with a value of $-0.152 \mathrm{mV}$. MHD voltages were zero at $0.0,0.5$, and 1.0 seconds. 